\title{
ROBIA: A Reaction Prediction Program
}

\author{
Ingrid M. Socorro, Keith Taylor, Jonathan M. Goodman \\ University Chemical Laboratory, Unilever Centre for Molecular Science Informatics, \\ University of Cambridge, Lensfield Road, Cambridge, CB2 1EW, UK, and Elsevier MDL, \\ 14600 Catalina Street, San Leandro, CA 94577, USA.
}

\section{Supporting Information}

\section{Table of contents}

S2 Table 1. All the structures generated by ROBIA during its application to investigate the proposed rearrangement to form Dolabriferol.

S11 Table 2. Energies for RHF/3-21G/water calculations and Monte Carlo searches using MM2. Number of structures minimized with good convergence $(s m g c)$ during the conformational search.

S16 Figure 1. Tree diagram of possible reaction pathways generated by ROBIA starting from linear precursor. 
Table 1. All the structures generated by ROBIA during its application to investigate
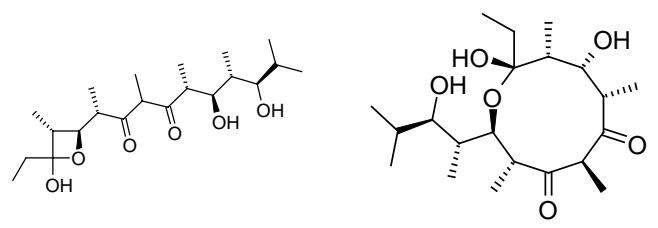

2<smiles>CC[C@H]1O[C@H]([C@@H](C)C(O)C(C)C)[C@@H](C)C(=O)C(C)C(=O)[C@H](C)[C@@H](O)[C@@H]1C</smiles>

5

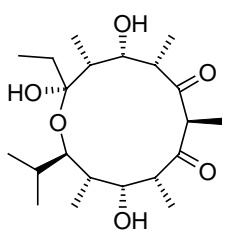

9

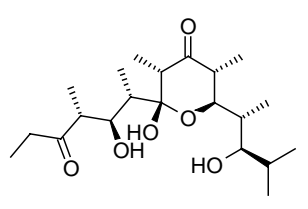

13

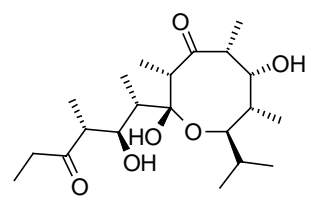

17

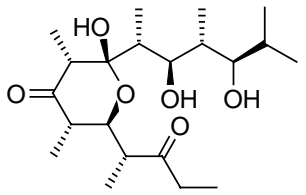

21

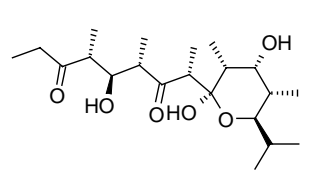

25

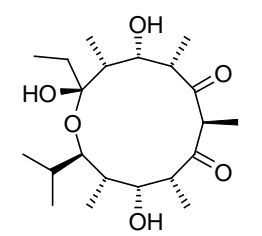

6

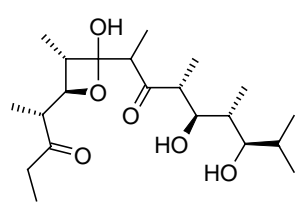

10

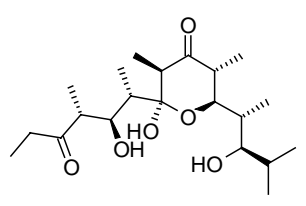

14

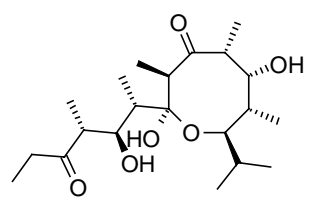

18

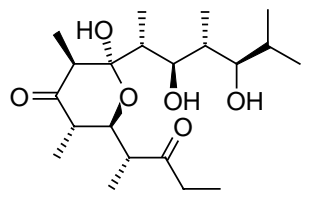

22

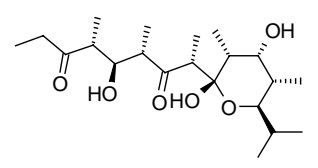

26

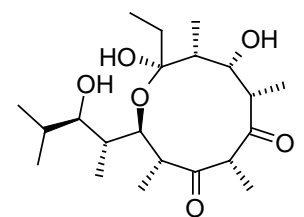

3

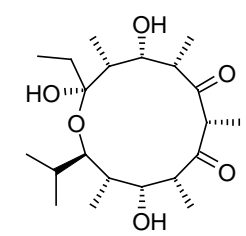

7

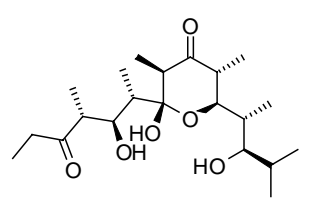

11

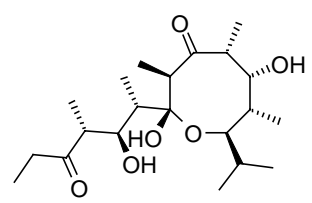

15

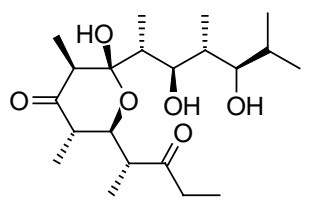

19

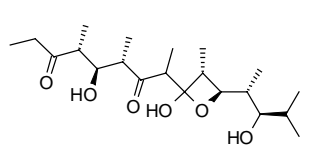

23

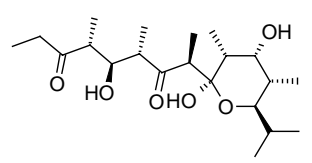

27

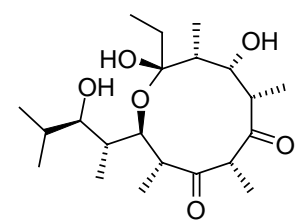

4

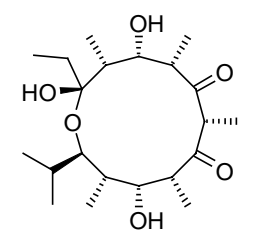

8

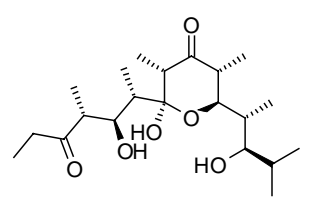

12

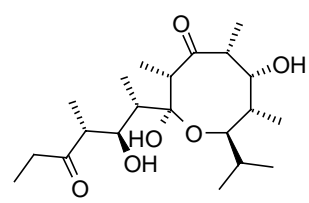

16

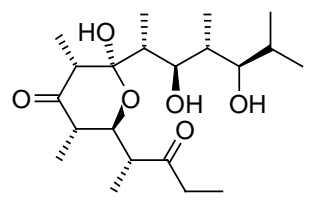

20

the proposed rearrangement to form Dolabriferol. 


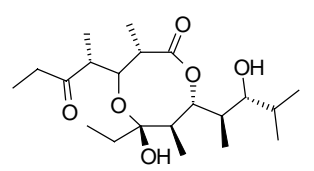

29

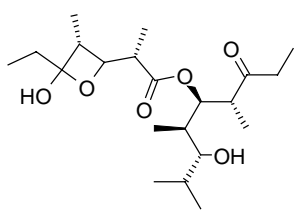

33

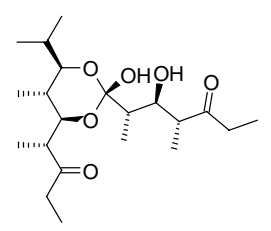

37

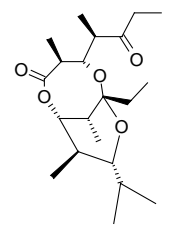

41

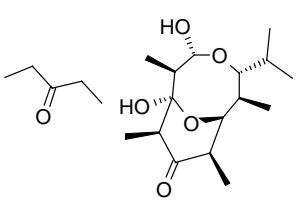

45a, 45b

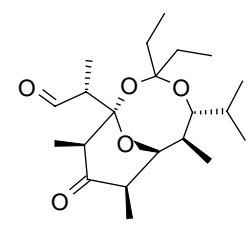

49

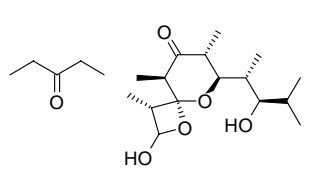

$53 a, 53 b$

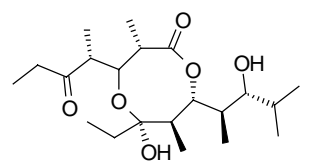

30

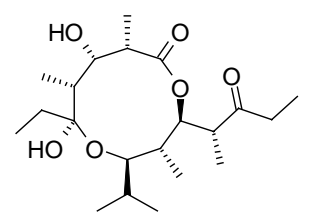

34

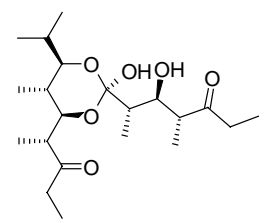

38

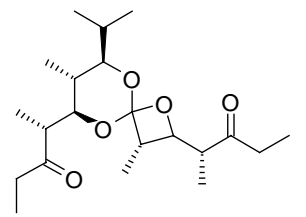

42

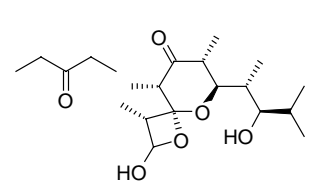

46a, 46b

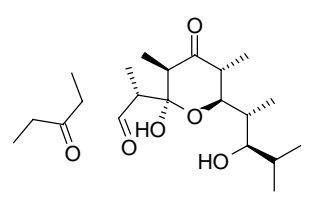

50a, 50b

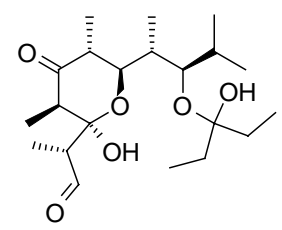

54

$\overbrace{\mathrm{O}}^{\left({ }_{\mathrm{OH}}\right.}$

31

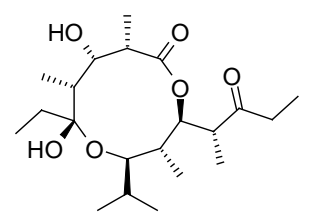

35

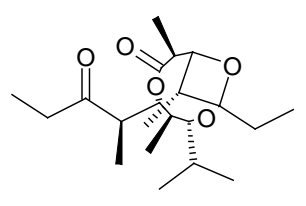

39

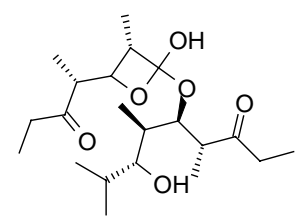

36

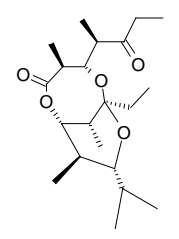

40

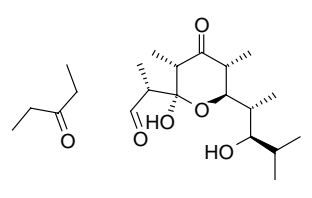

$43 a, 43 b$

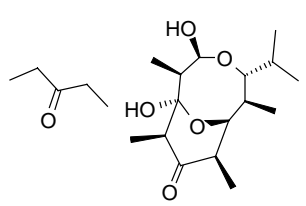

44a, 44b

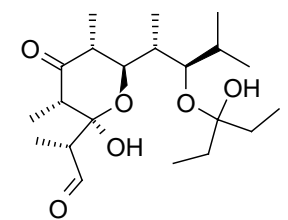

47

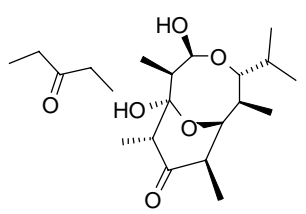

$51 \mathrm{a}, 51 \mathrm{~b}$

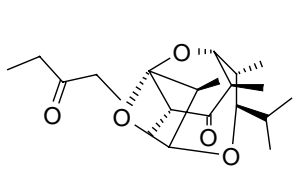

$55 a, 55 b$

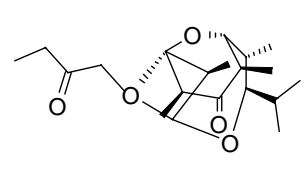

$48 a, 48 b$

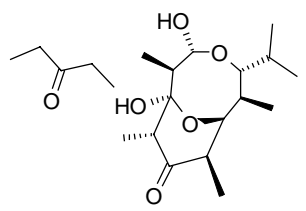

$52 \mathrm{a}, 52 \mathrm{~b}$

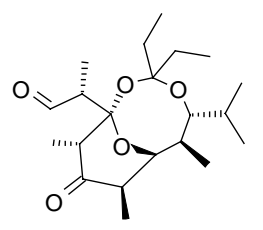

56 


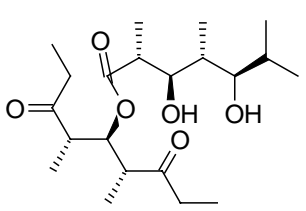

57

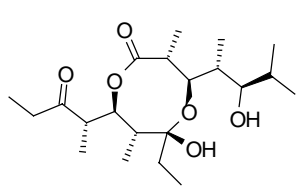

61

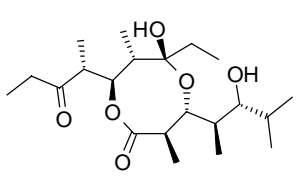

65

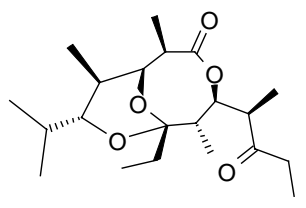

69

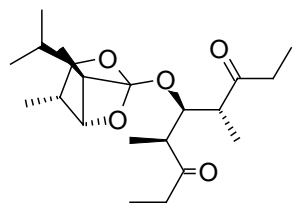

73

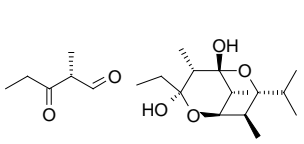

77a, 77b

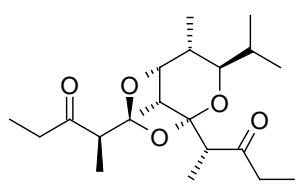

81

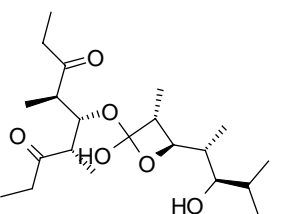

58

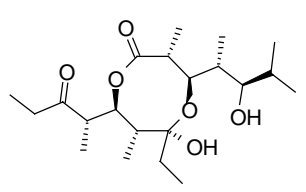

62

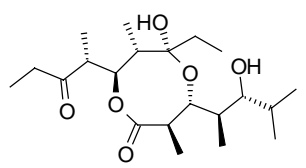

66

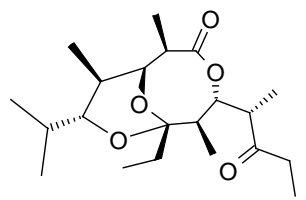

70

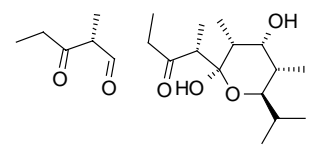

$74 a, 74 b$

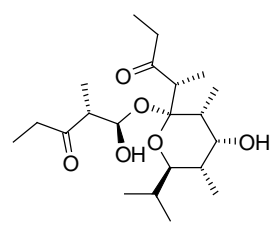

78

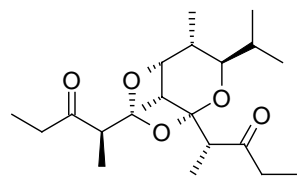

82

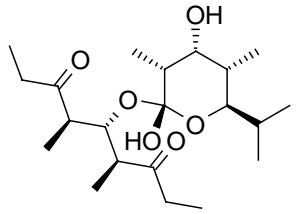

59

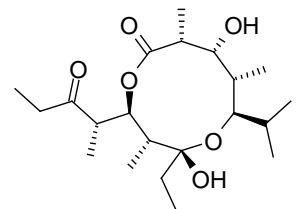

63

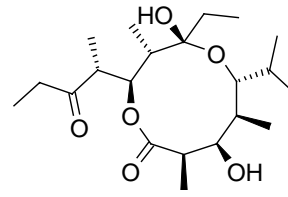

67

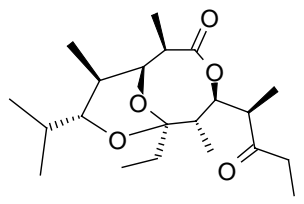

71

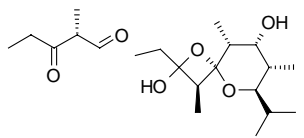

$75 a, 75 b$

$76 a, 76 b$

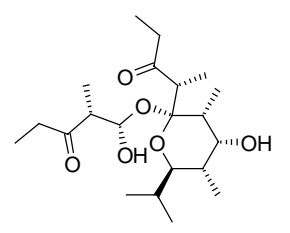

79

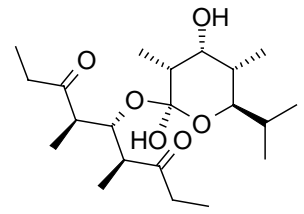

60

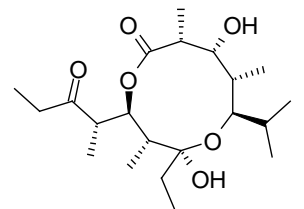

64

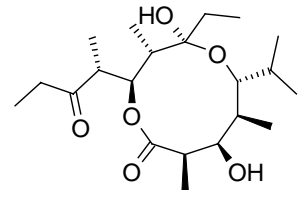

68

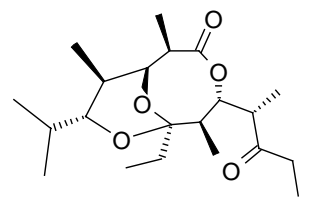

72
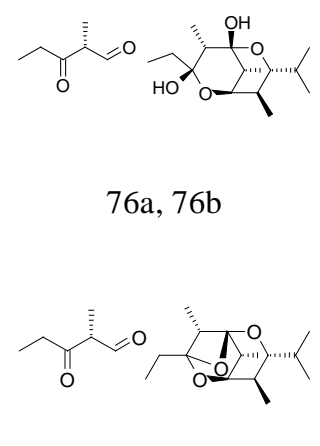

$80 a, 80 b$

$\prod_{\mathrm{O}}^{1}$

$83 a, 83 b$

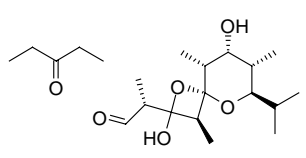

$84 a, 84 b$ 


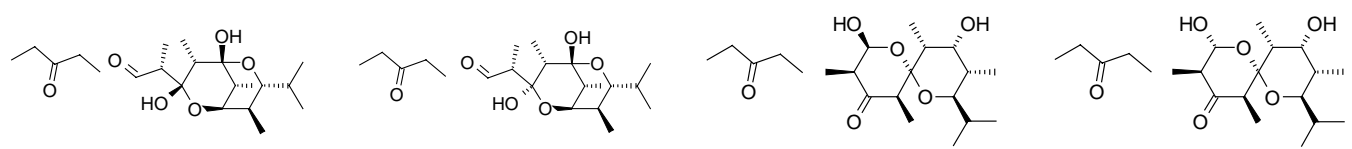

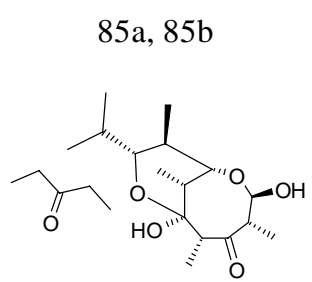

$89 a, 89 b$

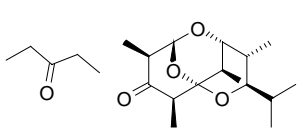

93a, 93b

$\overbrace{\mathrm{O}=\mathrm{i}}^{\overline{\bar{i}}=\mathrm{O}}$

97a, 97b

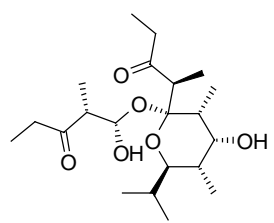

101

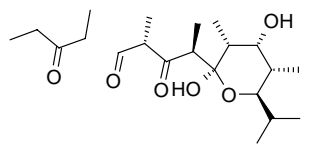

$105 a, 105 b$

$m_{O}^{2}$

109a, 109b
$86 a, 86 b$

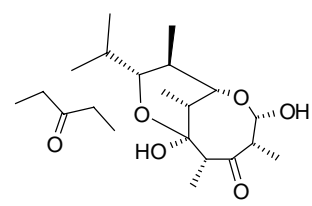

90a, 90b

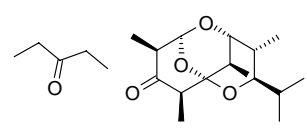

94a, 94b

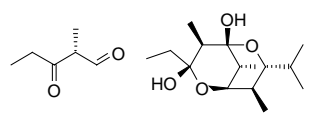

98a, 98b

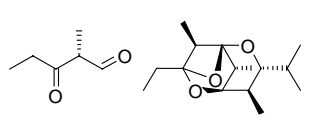

$102 a, 102 b$

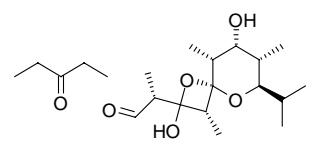

106a, 106b

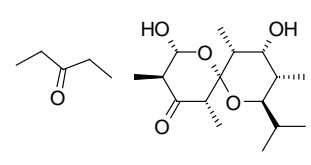

110a, 110b
$87 a, 87 b$

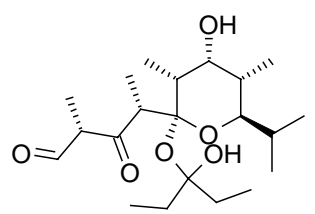

91

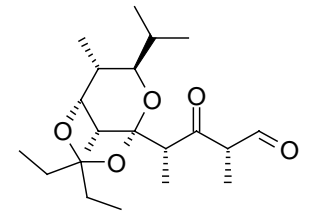

95

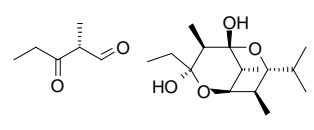

99a, 99b

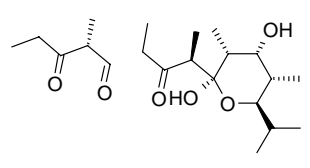

96a, 96b

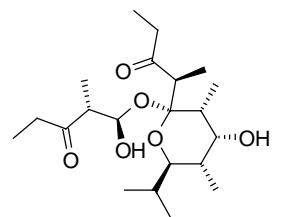

100

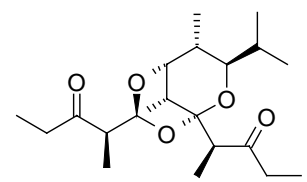

103

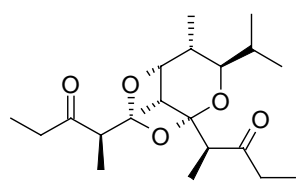

104

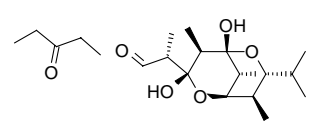

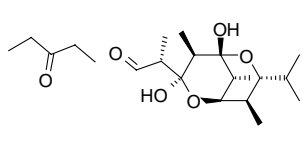

107a, 107b

108a, 108b

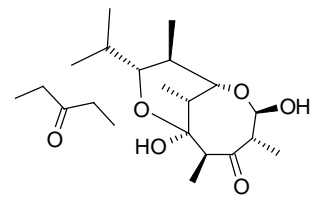

111a, 11b

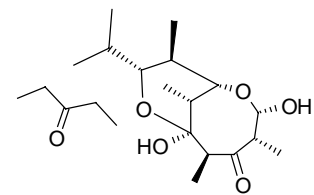

112a, $112 \mathrm{~b}$ 


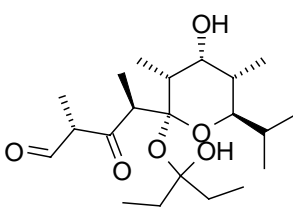

113

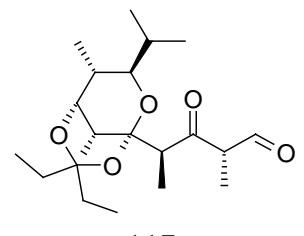

117<smiles>CCC(=O)[C@H](C)C1C[C@@H](O)[C@H](C)C2(O1)OC(C(C)C)[C@@H](C)C(=O)[C@H]2C</smiles>

121

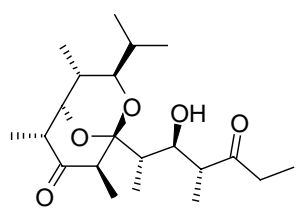

125

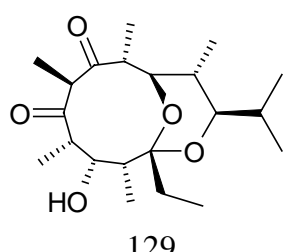

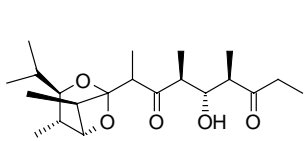

133

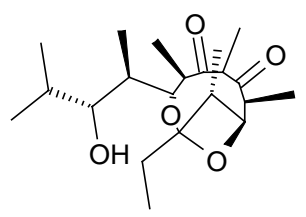

137

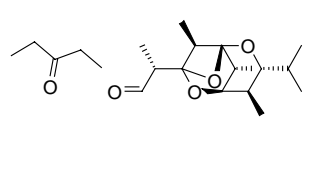

$114 a, 114 b$<smiles>CCC(=O)C(=O)C(C)[C@H](O)[C@H](C)C(=O)CC</smiles>

118a, 118b<smiles>CCC(=O)[C@H](C)C1O[C@]23OC(C(C)C)[C@@H](C)[C@H](O)[C@H]2C[C@H]3C(=O)[C@H]1C</smiles>

122

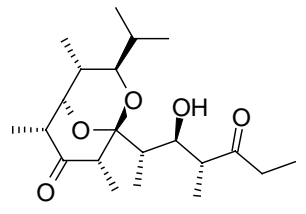

126
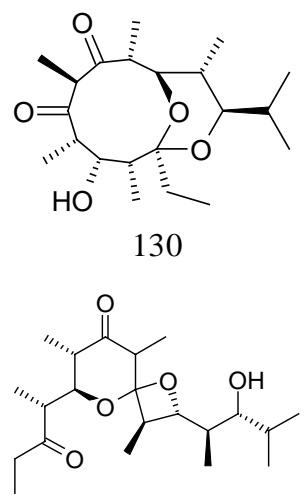

134

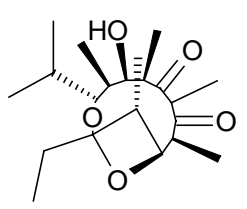

138

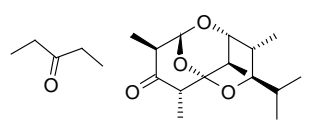

115a, 115b

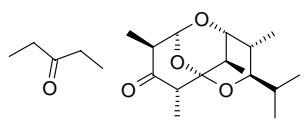

116a, 116b
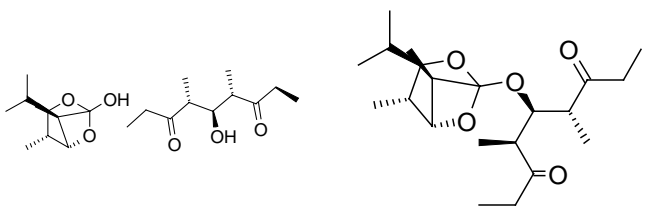

119a, 119b

120<smiles>CCC(=O)[C@H](C)C1O[C@]2(OC(C(C)C)[C@@H](C)C(=O)[C@H]2C)[C@@H](C)[C@@H](O)[C@@H]1C</smiles>

123<smiles>CCC(=O)[C@H](C)C1OC2(OC(C(C)C)[C@@H](C)[C@H](O)[C@H]2C)[C@@H](C)C(=O)[C@H]1C</smiles>

124

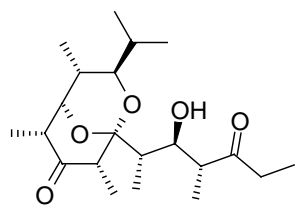

127

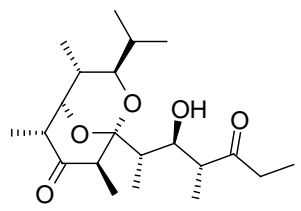

128
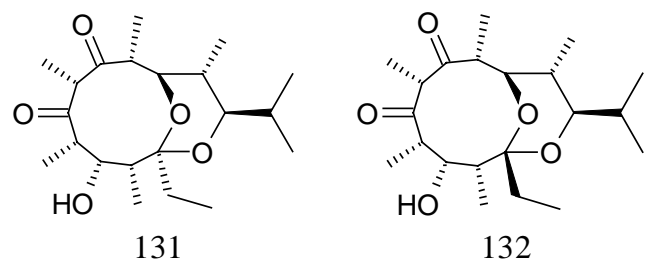

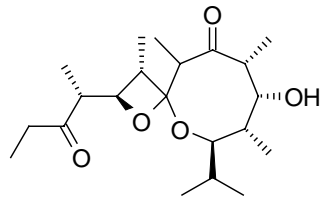

135

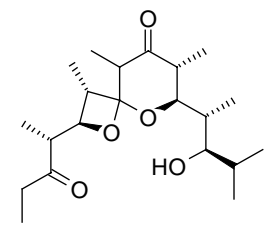

136

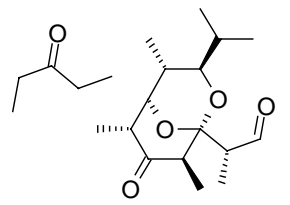

139a, 139b

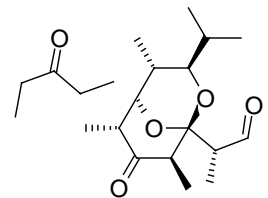

140a, 140b 


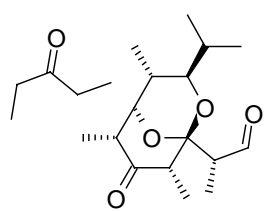

141a, 141b<smiles>CCC(=O)C(C)(C)C(=O)C(C)O[C@@]1(CC)OC(C(C)C)C(C)[C@@H]1C</smiles>

145

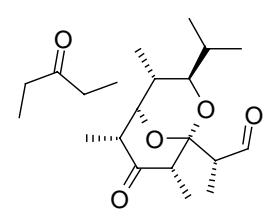

142a, 142b

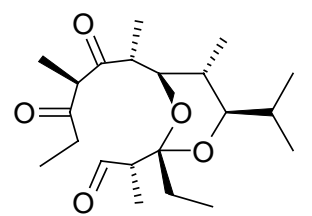

143

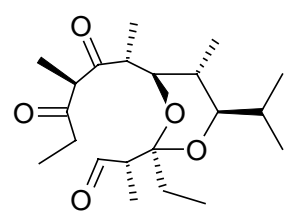

144

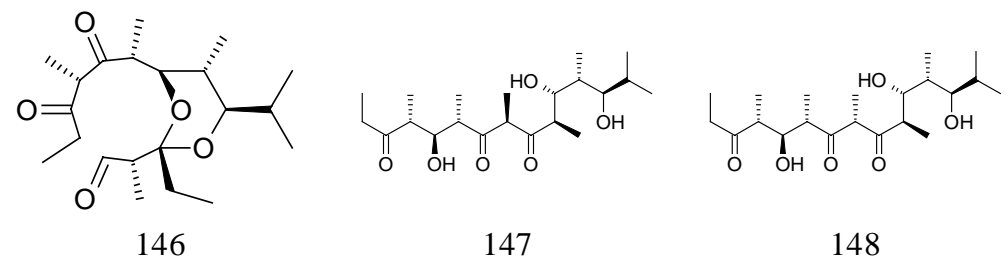

$\underbrace{\mathrm{O}}_{\mathrm{OH}} \underbrace{\mathrm{O}_{\mathrm{O}}}_{\mathrm{OH}}$<smiles>CCC(=O)C(C)C(O)C(C)C(O)C(C)(O)C(C)(O)C(C(C)C)C(C)C(C)C=O</smiles><smiles>CCC(=O)C(C)C(O)C(C)C(O)C(C)(O)C(C)(O)C(OC(C)C(C)C)C(C)C</smiles><smiles>CCC(=O)[C@H](C)[C@H]1OC(O)(CC)[C@@H](C)C(=O)[C@H]1C</smiles>

150

151<smiles>CCC(=O)[C@H](C)[C@H]1OC(O)(CC)C(C)C(=O)[C@H]1C</smiles>

153<smiles>CCC(=O)C(=O)C(C)C1(O)OC([C@H](C)C(=O)CC)[C@@H]1C</smiles>

154

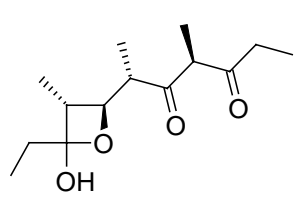

155
152<smiles>CCC(=O)[C@H](C)[C@H]1OC(OC(C(C)C)C(C)C=O)(OC2C(C)C(=O)C(C)C(=O)C2C)C(C)C1C</smiles>

156

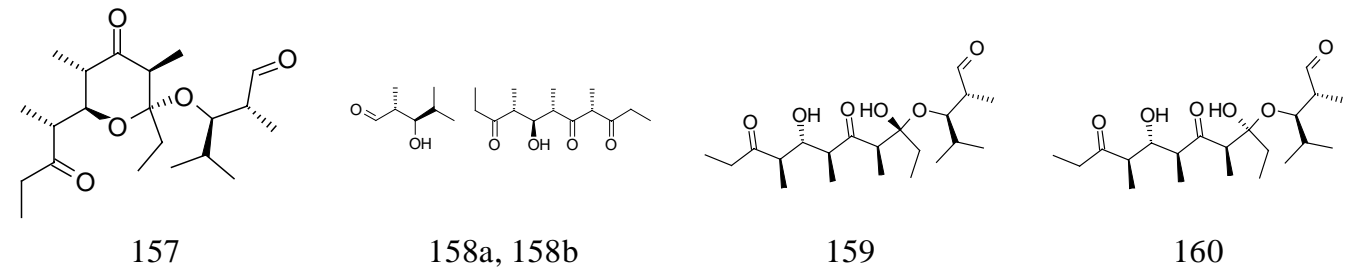<smiles>CCC(=O)[C@H](C)[C@H]1OC(O)(CC)[C@@H](C)C(=O)[C@H]1C</smiles>

161

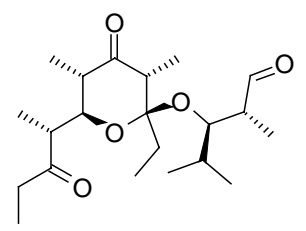

165<smiles>CCC(=O)C(C)[C@H]1OC(O)(CC)[C@@H](C)C(=O)[C@H]1C</smiles>

162<smiles>CCC(=O)C(=O)C(C)[C@H](C)[C@H](C)[C@@H]1OC(C)(O)[C@H]1C</smiles>

163

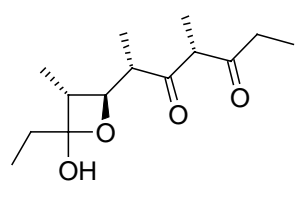

164<smiles>CCC(=O)[C@H](C)[C@H]1O[C@](C)(OC(C(C)C)C(C)C=O)[C@H](C)C(=O)[C@H]1C</smiles>

166<smiles>CCC(=O)CC(C)C(=O)CC(C)C(=O)C(C)C(C)C(C)C(C)C(C)C(C)C(C)C</smiles>

167a, 167b

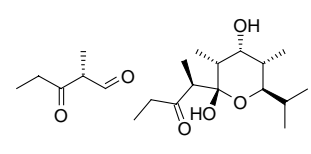

168a, 168b 


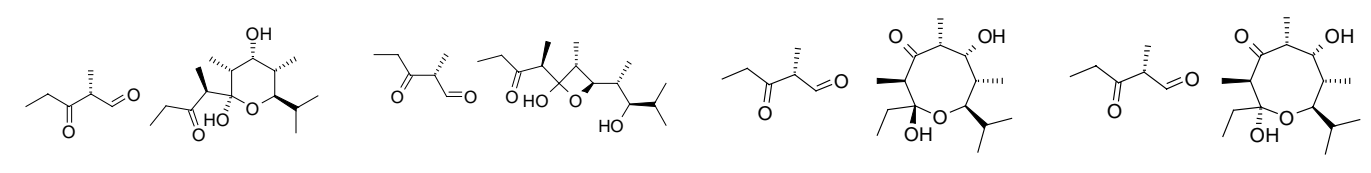

$169 a, 169 b$

170a, 170b

171a, 171b

$172 \mathrm{a}, 172 \mathrm{~b}$
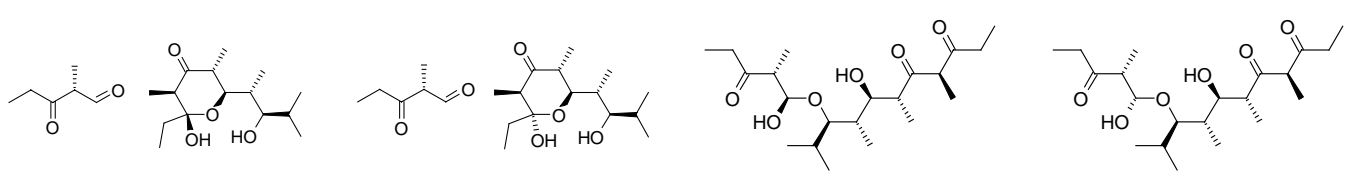

173a, 173b

174a, 174b

175

176

$\overbrace{0}^{=0}$

177a, 177b

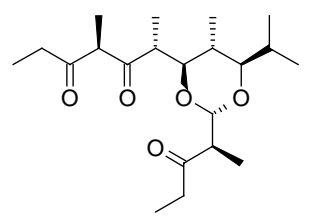

181

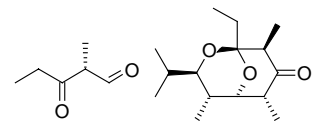

$178 \mathrm{a}, 178 \mathrm{~b}$

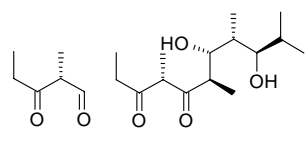

182a, $182 \mathrm{~b}$
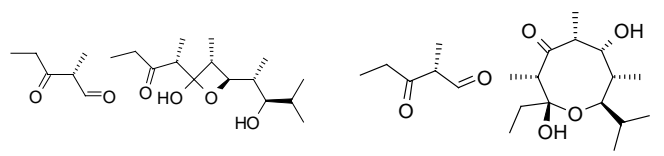

186a, 186b

185a, 185b

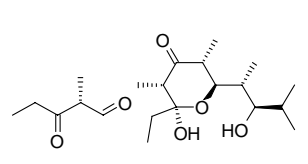

189a, 189b<smiles></smiles>

193a, 193b

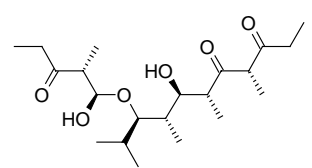

190

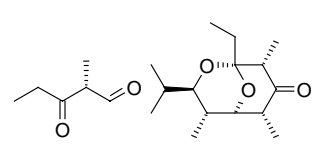

194a, 194b

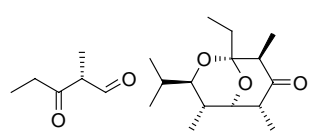

179a, 179b

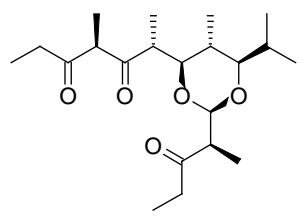

180

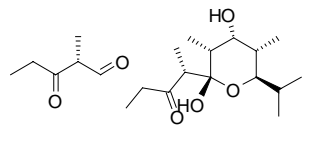

$183 a, 183 b$

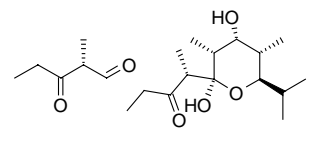

184a, 184b

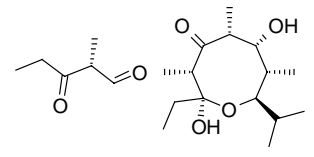

$187 \mathrm{a}, 187 \mathrm{~b}$

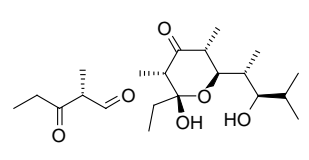

188a, 188b

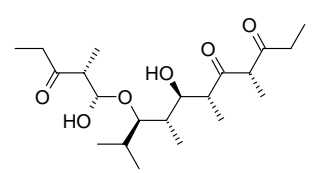

191

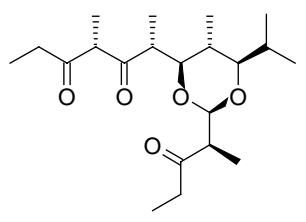

195

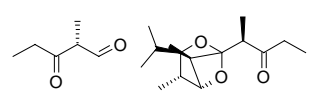

192a, 192b

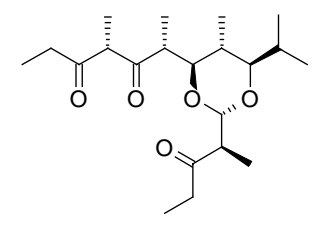

196 


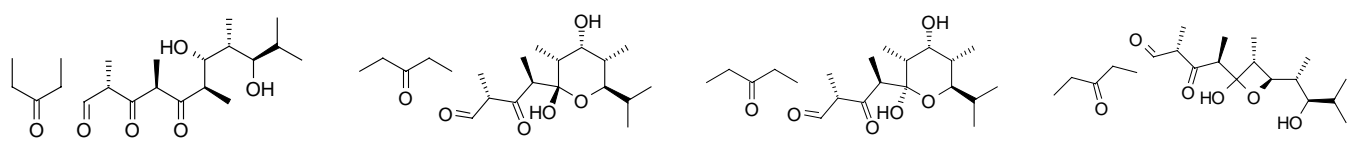

197a, 197b

198a, 198b

199a, 199b

200a, 200b<smiles>CCC(=O)CC[C@H](C)[C@@]1(O)OC(C(C)C)[C@@H](C)[C@@H](O)[C@H](C)C(=O)[C@H](C)[C@@H]1C</smiles><smiles>CCC(=O)CCC(C)[C@H]1OC(C(C)C)[C@@H](C)[C@H](O)[C@@H](C)C(=O)[C@H](C)[C@@H]1C</smiles>

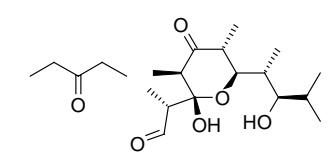<smiles>CCC(=O)CC(C)[C@H](C=O)[C@@]1(O)O[C@H]([C@H](C)C(O)C(C)C)[C@@H](C)C(=O)[C@H]1C</smiles>

201a, 201b

$202 a, 202 b$

203a, 203b

204a, 204b<smiles>CCCC(=O)CC</smiles><smiles>CCCC(=O)CC</smiles><smiles>CCC(=O)CO[C@@H]1C(O)O[C@H]([C@H](C)C(C)C)[C@H](C)[C@@H](C)C(=O)C1C</smiles><smiles>CCC(=O)COC1C(C)C(=O)[C@H](C)[C@@H](C(O)C(C)C)O[C@H]1[C@H](C)O</smiles>

205a, 205b

206a, 206b

207a, 207b

208a, 208b

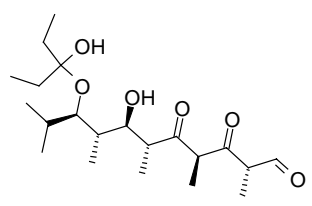

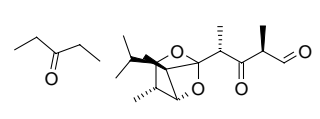<smiles>CCC(=O)CC</smiles><smiles>CCC(=O)CC</smiles>

209

210a, 210b

211a, 211b

212a, 212b<smiles>CCC(=O)CC</smiles>

213a, 213b<smiles>CCC(=O)CC[C@@H](C=O)C(=O)[C@H](C)[C@@]1(O)OC(C(C)C)[C@H](C)[C@H](O)[C@H]1C</smiles>

217a, 217b<smiles>CCC(=O)CCC(C)[C@H]1C(=O)[C@H](C)[C@@H](O)[C@@H](C)C(C(C)C)O[C@]1(O)C(C)C=O</smiles>

221a, 221b

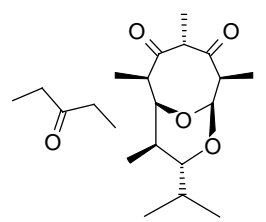

214a, 214b<smiles>CCC1(CC)OC(C(C)C)C(C)[C@@H](C(C)C(=O)C(C)C(=O)C(C)C=O)O1</smiles>

215

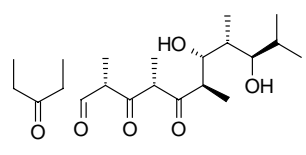

216a, 216b

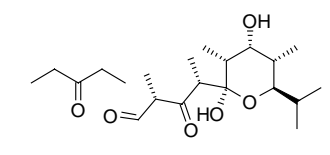

218a, 218b

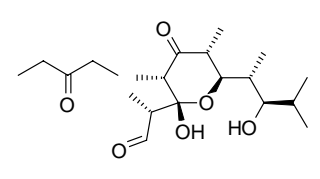

222a, 222b

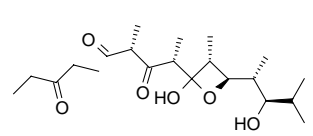

219a, 219b

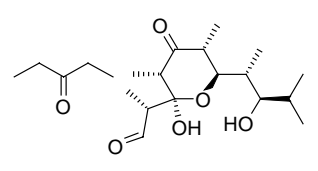

223a, 223b

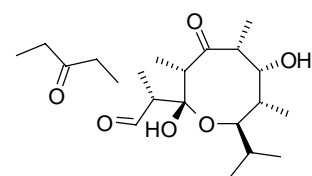

220a, 220b

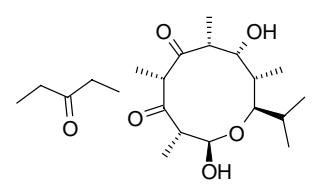

224a, 224b 


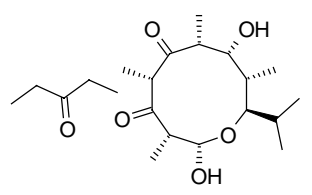

225a, 225b

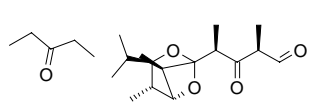

229a, 229b

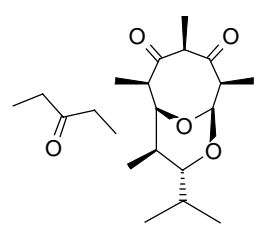

233a, 233b

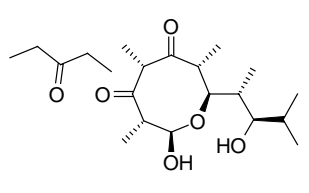

226a, 226b

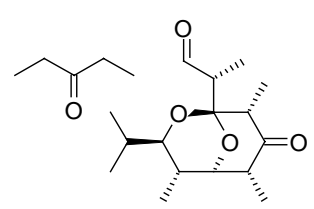

230a, 230b

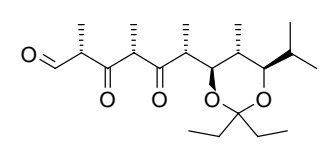

234

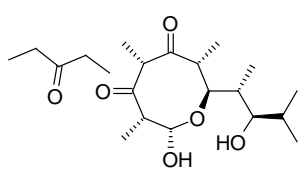

227a, 227b

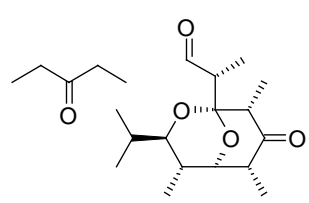

231a, 231b
228
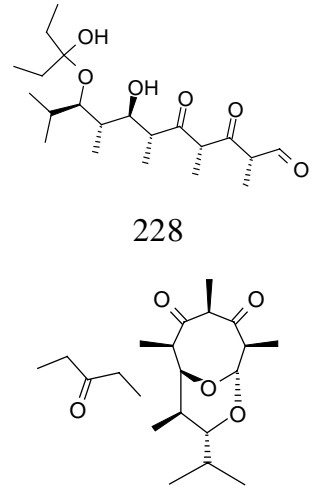

232a, 232b 
Table2. Energies for RHF/3-21G/water calculations and Monte Carlo searches using MM2. Number of structures minimized with good convergence $(s m g c)$ during the conformational search. (No calculations were done on the structures filtered by the rule-based filters)

\begin{tabular}{|c|c|c|c|}
\hline Structure \# & $\begin{array}{c}\text { RHF/3-21G/water } \\
\text { (hartrees) }\end{array}$ & MM2 (kJ/mol) & Number of $s m g c$ \\
\hline 1 & $\mathrm{f}^{\mathrm{i}}$ & $\mathrm{f}$ & $\mathrm{f}$ \\
\hline 2 & $\mathrm{f}$ & -37.72886 & 1943 \\
\hline 3 & $\mathrm{f}$ & -63.84457 & 1093 \\
\hline 4 & $\mathrm{f}$ & -46.82077 & 1488 \\
\hline 5 & $\mathrm{f}$ & -53.45810 & 1653 \\
\hline 6 & $\mathrm{f}$ & -31.25841 & 1329 \\
\hline 7 & $\mathrm{f}$ & -65.35514 & 437 \\
\hline 8 & $\mathrm{f}$ & -42.27930 & 938 \\
\hline 9 & $\mathrm{f}$ & -52.64730 & 749 \\
\hline 10 & $\mathrm{f}$ & $\mathrm{f}$ & $\mathrm{f}$ \\
\hline 11 & $\mathrm{f}$ & -70.67517 & 1745 \\
\hline 12 & -1259.53594607811 & -73.50029 & 2519 \\
\hline 13 & $\mathrm{f}$ & -58.02185 & 2117 \\
\hline 14 & -1259.54139964144 & -81.15226 & 1399 \\
\hline 15 & $\mathrm{f}$ & -5.054635 & 1424 \\
\hline 16 & $\mathrm{f}$ & -23.82581 & 2035 \\
\hline 17 & $\mathrm{f}$ & -2.914771 & 1191 \\
\hline 18 & $\mathrm{f}$ & -28.82405 & 1274 \\
\hline 19 & -1259.53983298539 & -74.53102 & 1796 \\
\hline 20 & -1259.54043421725 & -81.08069 & 2115 \\
\hline 21 & $\mathrm{f}$ & -62.07300 & 2209 \\
\hline 22 & -1259.53662106082 & -83.85850 & 1520 \\
\hline 23 & $\mathrm{f}$ & $\mathrm{f}$ & $\mathrm{f}$ \\
\hline 24 & $\mathrm{f}$ & -70.38535 & 1914 \\
\hline 25 & -1259.55014130661 & -93.60656 & 2013 \\
\hline 26 & $\mathrm{f}$ & -67.92147 & 2061 \\
\hline 27 & -1259.5475753903 & -95.71644 & 1911 \\
\hline 28 & -1259.5481529915 & 127.3708 & 2600 \\
\hline 29 & -1259.52809307898 & 173.5322 & 677 \\
\hline 30 & -1259.53134882983 & 155.3954 & 833 \\
\hline 31 & -1259.55481387218 & 88.47402 & 1782 \\
\hline 32 & -1259.54937918969 & 71.25047 & 1818 \\
\hline 33 & $\mathrm{f}$ & $\mathrm{f}$ & $\mathrm{f}$ \\
\hline 34 & -1259.52857595088 & 146.4399 & 615 \\
\hline 35 & -1259.53186849242 & 158.9727 & 792 \\
\hline 36 & $\mathrm{f}$ & $\mathrm{f}$ & $\mathrm{f}$ \\
\hline 37 & -1259.549708062 & -306.8563 & 287 \\
\hline 38 & -1259.55840987696 & -329.5379 & 339 \\
\hline 39 & $\mathrm{f}$ & $\mathrm{f}$ & $\mathrm{f}$ \\
\hline 40 & -1183.79182172119 & 400.7116 & 31 \\
\hline 41 & -1183.90965494857 & 133.2561 & 194 \\
\hline 42 & $\mathrm{f}$ & $\mathrm{f}$ & $\mathrm{f}$ \\
\hline $43 a^{\mathrm{ii}}$ & -268.5320931907 & 9.171217 & 6 \\
\hline
\end{tabular}

\footnotetext{
${ }^{\mathrm{i}} \mathrm{f}$ indicates that the structures were filtered by rule-based filters or based on MM2 results

${ }^{\text {ii }}$ Fragment 43a also represents 44a-46a, 48a, 50a-53a, 55a, 83a-90a, 92a-94a, 105a-112a, 114a-116a, 139a, 142a, 197a-208a, 210a-214a, 216a-227a, 229a-233a, as they are the same molecule
} 


\begin{tabular}{|c|c|c|c|}
\hline $43 b$ & -990.99902345117 & -116.6008 & 1271 \\
\hline $44 b$ & -990.97708828948 & -57.75555 & 232 \\
\hline $45 b$ & -990.98084771698 & -64.00031 & 175 \\
\hline $46 a, 46 b$ & $\mathrm{f}$ & $\mathrm{f}$ & $\mathrm{f}$ \\
\hline 47 & -1259.52274956431 & -161.3217 & 944 \\
\hline $48 \mathrm{a}, 48 \mathrm{~b}$ & $\mathrm{f}$ & $\mathrm{f}$ & $\mathrm{f}$ \\
\hline 49 & -1183.88123175685 & -166.6435 & 497 \\
\hline $50 \mathrm{~b}$ & -990.99340482451 & -117.2908 & 1463 \\
\hline $51 b$ & -990.97704094623 & -57.75557 & 233 \\
\hline $52 \mathrm{~b}$ & -990.97993594835 & -64.00024 & 156 \\
\hline $53 a, 53 b$ & $\mathrm{f}$ & $\mathrm{f}$ & $\mathrm{f}$ \\
\hline 54 & -1259.52395492611 & -167.0971 & 707 \\
\hline $55 \mathrm{a}, 55 \mathrm{~b}$ & $\mathrm{f}$ & f & $\mathrm{f}$ \\
\hline 56 & -1183.18195169756 & 1082.676 & 305 \\
\hline 57 & -1259.53662106082 & 163.4709 & 1850 \\
\hline 58 & $\mathrm{f}$ & $\mathrm{f}$ & f \\
\hline 59 & -1259.54237664407 & -291.4636 & 513 \\
\hline 60 & -1259.55745639727 & -303.7664 & 368 \\
\hline 61 & -1259.51435136116 & 204.7549 & 1015 \\
\hline 62 & -1259.51680822157 & 184.3267 & 962 \\
\hline 63 & -1259.51558778021 & 155.5832 & 880 \\
\hline 64 & -1259.51448732298 & 156.3335 & 839 \\
\hline 65 & -1259.54460530581 & 149.4317 & 721 \\
\hline 66 & -1259.53295211056 & 177.8018 & 787 \\
\hline 67 & -1259.53648360156 & 141.7967 & 685 \\
\hline 68 & -1259.53880852674 & 156.0340 & 1002 \\
\hline 69 & -1183.92656977354 & 125.4563 & 971 \\
\hline 70 & -1183.89820218555 & 186.4975 & 492 \\
\hline 71 & -1183.92652634513 & 125.4576 & 579 \\
\hline 72 & -1183.91164213261 & 154.6343 & 578 \\
\hline 73 & $\mathrm{f}$ & $\mathrm{f}$ & $\mathrm{f}$ \\
\hline $74 a^{\mathrm{iii}}$ & -380.61815733808 & -66.94791 & 26 \\
\hline $74 b$ & -878.91160174485 & -70.06717 & 332 \\
\hline $75 a, 75 b$ & $\mathrm{f}$ & $\mathrm{f}$ & $\mathrm{f}$ \\
\hline $76 a, 76 b$ & $\mathrm{f}$ & $\mathrm{f}$ & $\mathrm{f}$ \\
\hline $77 a, 77 b$ & $\mathrm{f}$ & $\mathrm{f}$ & $\mathrm{f}$ \\
\hline 78 & -1259.54843025099 & -245.5561 & 312 \\
\hline 79 & -1259.54845146156 & -245.5549 & 346 \\
\hline $80 \mathrm{a}, 80 \mathrm{~b}$ & $\mathrm{f}$ & $\mathrm{f}$ & $\mathrm{f}$ \\
\hline 81 & -1183.91782046676 & -237.4271 & 1717 \\
\hline 82 & -1183.92819761018 & -251.5348 & 1352 \\
\hline $83 b$ & -990.99759655458 & -147.8432 & 648 \\
\hline $84 a, 84 b$ & $\mathrm{f}$ & $\mathrm{f}$ & $\mathrm{f}$ \\
\hline $85 a, 85 b$ & $\mathrm{f}$ & $\mathrm{f}$ & $\mathrm{f}$ \\
\hline $86 a, 86 b$ & $\mathrm{f}$ & $\mathrm{f}$ & $\mathrm{f}$ \\
\hline $87 b$ & -990.99598688843 & -216.2332 & 163 \\
\hline $88 b$ & -991.00541220436 & -225.6064 & 105 \\
\hline $89 b$ & -990.97780418167 & -98.03250 & 49 \\
\hline $90 b$ & -990.98202794719 & -85.17113 & 71 \\
\hline
\end{tabular}

\footnotetext{
iii Fragment 74a also represents 75a-77a, 80a, 96a-99a, 102a, 167a-174a, 177a-179a, 183a-189a, 192a$194 \mathrm{a}$, as they are the same molecule
} 


\begin{tabular}{|c|c|c|c|}
\hline 91 & -1259.51113186137 & -236.2788 & 783 \\
\hline $92 a, 92 b$ & $\mathrm{f}$ & $\mathrm{f}$ & $\mathrm{f}$ \\
\hline $93 a, 93 b$ & $\mathrm{f}$ & $\mathrm{f}$ & $\mathrm{f}$ \\
\hline $94 b$ & -915.22291789938 & 23.13076 & 11 \\
\hline 95 & -1183.16481983112 & 742.9153 & 606 \\
\hline $96 b$ & -878.91202757283 & -77.41107 & 304 \\
\hline $97 a, 97 b$ & $\mathrm{f}$ & $\mathrm{f}$ & $\mathrm{f}$ \\
\hline $98 \mathrm{a}, 98 \mathrm{~b}$ & $\mathrm{f}$ & $\mathrm{f}$ & $\mathrm{f}$ \\
\hline $99 a, 99 b$ & $\mathrm{f}$ & $\mathrm{f}$ & $\mathrm{f}$ \\
\hline 100 & -1259.53492100543 & -239.9560 & 399 \\
\hline 101 & -1259.54636178912 & -243.6361 & 405 \\
\hline $102 a, 102 b$ & $\mathrm{f}$ & $\mathrm{f}$ & $\mathrm{f}$ \\
\hline 103 & -1183.92208025774 & -242.9996 & 1501 \\
\hline 104 & -1183.92729411659 & -253.3482 & 1282 \\
\hline $105 \mathrm{~b}$ & -990.99561069987 & -150.9094 & 693 \\
\hline $106 a, 106 b$ & $\mathrm{f}$ & $\mathrm{f}$ & $\mathrm{f}$ \\
\hline $107 a, 107 b$ & $\mathrm{f}$ & $\mathrm{f}$ & $\mathrm{f}$ \\
\hline $108 a, 108 b$ & $\mathrm{f}$ & $\mathrm{f}$ & $\mathrm{f}$ \\
\hline $109 b$ & -991.00393684755 & -222.5773 & 168 \\
\hline $110 \mathrm{~b}$ & -991.00556653965 & -222.5773 & 170 \\
\hline $111 b$ & -990.97048073168 & -207.1604 & 102 \\
\hline $112 \mathrm{~b}$ & -990.97280629575 & -68.26826 & 167 \\
\hline 113 & -1259.53336315953 & -225.7639 & 452 \\
\hline $114 a, 114 b$ & $\mathrm{f}$ & $\mathrm{f}$ & $\mathrm{f}$ \\
\hline $115 a, 115 b$ & $\mathrm{f}$ & $\mathrm{f}$ & $\mathrm{f}$ \\
\hline $116 \mathrm{~b}$ & -915.22533207345 & 15.79115 & 5 \\
\hline 117 & -1183.90047132183 & -243.0374 & 1472 \\
\hline $118 \mathrm{a}$ & -649.17224461114 & -9.857260 & 362 \\
\hline $118 b$ & -610.36476400387 & 176.9717 & 55 \\
\hline $119 a, 119 b$ & $\mathrm{f}$ & $\mathrm{f}$ & $\mathrm{f}$ \\
\hline 120 & $\mathrm{f}$ & $\mathrm{f}$ & $\mathrm{f}$ \\
\hline 121 & -1183.94675015652 & -117.6571 & 360 \\
\hline 122 & -1183.92777116589 & -84.44629 & 553 \\
\hline 123 & -1183.93060927891 & -95.35584 & 533 \\
\hline 124 & -1183.92738867476 & -101.7484 & 607 \\
\hline 125 & -1183.84623135345 & 54.85320 & 560 \\
\hline 126 & -1183.84153967195 & 68.97997 & 615 \\
\hline 127 & -1183.82677478525 & 98.22806 & 492 \\
\hline 128 & -1183.83694772099 & 82.28378 & 449 \\
\hline 129 & -1183.90201876627 & -63.72773 & 857 \\
\hline 130 & -1183.92190122801 & -104.9614 & 422 \\
\hline 131 & -1183.92006731389 & -103.2263 & 566 \\
\hline 132 & -1183.90845215189 & -75.07350 & 534 \\
\hline 133 & $\mathrm{f}$ & $\mathrm{f}$ & $\mathrm{f}$ \\
\hline 134 & $\mathrm{f}$ & $\mathrm{f}$ & $\mathrm{f}$ \\
\hline 135 & $\mathrm{f}$ & $\mathrm{f}$ & $\mathrm{f}$ \\
\hline 136 & $\mathrm{f}$ & $\mathrm{f}$ & $\mathrm{f}$ \\
\hline 137 & $\mathrm{f}$ & $\mathrm{f}$ & $\mathrm{f}$ \\
\hline 138 & $\mathrm{f}$ & $\mathrm{f}$ & $\mathrm{f}$ \\
\hline
\end{tabular}




\begin{tabular}{|c|c|c|c|}
\hline $139 b$ & -915.29902812876 & 11.62042 & 24 \\
\hline $140 \mathrm{~b}$ & -915.29250425177 & 19.39330 & 24 \\
\hline $141 \mathrm{~b}$ & -915.29354678137 & 19.39327 & 24 \\
\hline $142 b$ & -915.29252071516 & 19.74944 & 25 \\
\hline 143 & -1183.91193688556 & -181.1746 & 456 \\
\hline 144 & -1183.91107948181 & -194.3528 & 465 \\
\hline 145 & -1183.91053410198 & -193.7070 & 409 \\
\hline 146 & -1183.90859121259 & -181.0131 & 483 \\
\hline 147 & -1259.52825914931 & -70.38560 & 2228 \\
\hline 148 & -1259.52792140708 & -81.59570 & 1399 \\
\hline $149 a^{\text {iv }}$ & -420.6026652107 & 0.4754602 & 71 \\
\hline $149 \mathrm{~b}$ & -838.91256530498 & -76.95892 & 1732 \\
\hline 150 & -1259.51577186882 & -102.4777 & 167 \\
\hline 151 & -1259.51582755858 & -116.1555 & 160 \\
\hline 152 & -838.92626241963 & -106.7202 & 361 \\
\hline 153 & -838.92689258255 & -106.7203 & 361 \\
\hline 154 & $\mathrm{f}$ & $\mathrm{f}$ & $\mathrm{f}$ \\
\hline 155 & $\mathrm{f}$ & $\mathrm{f}$ & $\mathrm{f}$ \\
\hline 156 & -1183.90813545509 & -122.7233 & 1302 \\
\hline 157 & -1183.91237131097 & -119.6830 & 713 \\
\hline $158 \mathrm{~b}$ & -838.91670653279 & -74.76302 & 494 \\
\hline 159 & -1259.52124456577 & -116.4926 & 153 \\
\hline 160 & -1259.51015767901 & -110.3652 & 148 \\
\hline 161 & -838.92523894099 & -104.5793 & 288 \\
\hline 162 & -838.92459930191 & -104.5793 & 283 \\
\hline 163 & $\mathrm{f}$ & $\mathrm{f}$ & $\mathrm{f}$ \\
\hline 164 & $\mathrm{f}$ & $\mathrm{f}$ & $\mathrm{f}$ \\
\hline 165 & -1183.91168677264 & -126.4967 & 680 \\
\hline 166 & -1183.91164125782 & -126.4881 & 674 \\
\hline $167 \mathrm{~b}$ & -878.88494516165 & -40.97802 & 2761 \\
\hline $168 \mathrm{~b}$ & -878.89385816736 & -45.78718 & 581 \\
\hline $169 b$ & -878.91206276502 & -77.41103 & 271 \\
\hline $170 a, 170 b$ & $\mathrm{f}$ & f & $\mathrm{f}$ \\
\hline $171 b$ & -878.88013819564 & -7.528426 & 779 \\
\hline $172 b$ & -878.88295470609 & -26.48803 & 480 \\
\hline $173 b$ & -878.89547706263 & -57.52542 & 1283 \\
\hline $174 \mathrm{~b}$ & -878.90027519896 & -62.89773 & 1082 \\
\hline 175 & -1259.52397397318 & -158.9396 & 66 \\
\hline 176 & -1259.53037085326 & -161.7985 & 82 \\
\hline $177 a, 177 b$ & $\mathrm{f}$ & $\mathrm{f}$ & $\mathrm{f}$ \\
\hline $178 \mathrm{~b}$ & -803.20610417126 & 53.64252 & 10 \\
\hline $179 a, 179 b$ & $\mathrm{f}$ & $\mathrm{f}$ & $\mathrm{f}$ \\
\hline 180 & -1183.91982146291 & -218.1615 & 252 \\
\hline 181 & -1183.92669067347 & -204.6759 & 253 \\
\hline $182 \mathrm{~b}$ & -878.88767706717 & -43.60885 & 2611 \\
\hline $183 \mathrm{~b}$ & -878.89568985982 & -39.23885 & 857 \\
\hline $184 b$ & -878.91161828782 & -70.06720 & 344 \\
\hline $185 a, 185 b$ & $\mathrm{f}$ & f & $\mathrm{f}$ \\
\hline $186 \mathrm{~b}$ & -878.87368851191 & -7.064808 & 549 \\
\hline
\end{tabular}

\footnotetext{
${ }^{\text {iv }}$ Fragment $149 \mathrm{a}$ also represents 158 a, as they are the same molecule
} 


\begin{tabular}{|c|c|c|c|}
\hline $187 \mathrm{~b}$ & -878.89018197844 & -26.58895 & 408 \\
\hline $188 b$ & -878.89324110274 & -54.36880 & 1117 \\
\hline $189 \mathrm{~b}$ & -878.89883502552 & -69.53603 & 933 \\
\hline 190 & -1259.52354580144 & -159.6780 & 88 \\
\hline 191 & -1259.52955810479 & -156.4727 & 92 \\
\hline $192 a, 192 b$ & $\mathrm{f}$ & $\mathrm{f}$ & $\mathrm{f}$ \\
\hline $193 b$ & -803.2052398053 & 58.10770 & 9 \\
\hline $194 a, 194 b$ & $\mathrm{f}$ & $\mathrm{f}$ & $\mathrm{f}$ \\
\hline 195 & -1183.92327810984 & -215.8949 & 292 \\
\hline 196 & -1183.92544006206 & -202.7607 & 289 \\
\hline $197 b$ & -990.97246967264 & -117.5746 & 3209 \\
\hline $198 b$ & -990.97771360055 & -120.5758 & 920 \\
\hline $199 b$ & -990.99550445617 & -150.9094 & 582 \\
\hline $200 \mathrm{a}, 200 \mathrm{~b}$ & $\mathrm{f}$ & $\mathrm{f}$ & $\mathrm{f}$ \\
\hline $201 b$ & -990.96850761638 & -56.77705 & 511 \\
\hline $202 b$ & -990.97251506199 & -64.95137 & 800 \\
\hline $203 b$ & -990.98899352034 & -116.4087 & 680 \\
\hline $204 b$ & -990.99420417784 & -117.2908 & 1149 \\
\hline $205 b$ & -990.97603134833 & -112.2158 & 468 \\
\hline $206 b$ & -990.98079748268 & -112.4664 & 426 \\
\hline $207 b$ & -990.97660912137 & -101.6512 & 1211 \\
\hline $208 b$ & -990.97589593803 & -101.6522 & 1188 \\
\hline 209 & -1259.51326607047 & -170.0222 & 179 \\
\hline $210 \mathrm{a}, 210 \mathrm{~b}$ & $\mathrm{f}$ & $\mathrm{f}$ & $\mathrm{f}$ \\
\hline $211 \mathrm{~b}$ & -915.38875396285 & -156.8975 & 48 \\
\hline $212 a, 212 b$ & $\mathrm{f}$ & $\mathrm{f}$ & $\mathrm{f}$ \\
\hline $213 b$ & -915.37605575503 & -135.0569 & 40 \\
\hline $214 b$ & -915.36748031697 & -126.4919 & 37 \\
\hline 215 & -1183.90836183758 & -222.9952 & 851 \\
\hline $216 b$ & -990.97361383683 & -128.1420 & 807 \\
\hline $217 b$ & -990.98458022531 & -114.7582 & 1244 \\
\hline $218 b$ & -990.99745754934 & -147.8432 & 620 \\
\hline $219 a, 219 b$ & $\mathrm{f}$ & $\mathrm{f}$ & $\mathrm{f}$ \\
\hline $220 b$ & -990.96010075577 & -49.22660 & 640 \\
\hline $221 b$ & -990.97594918308 & -69.45309 & 674 \\
\hline $222 b$ & -990.9799330564 & -100.0589 & 1271 \\
\hline $223 b$ & -990.99882097575 & -116.6003 & 1297 \\
\hline $224 b$ & -990.98505133314 & -115.4959 & 352 \\
\hline $225 b$ & -990.98742548241 & -122.7934 & 218 \\
\hline $226 b$ & -990.97945522154 & -108.3431 & 959 \\
\hline $227 b$ & -990.97928460015 & -108.3434 & 1005 \\
\hline 228 & -1259.50686258161 & -171.4594 & 161 \\
\hline $229 a, 229 b$ & $\mathrm{f}$ & $\mathrm{f}$ & $\mathrm{f}$ \\
\hline $230 \mathrm{~b}$ & -915.37930932635 & -141.7106 & 63 \\
\hline $231 \mathrm{a}, 231 \mathrm{~b}$ & $\mathrm{f}$ & f & $\mathrm{f}$ \\
\hline $232 b$ & -915.37566773129 & -135.1228 & 39 \\
\hline $233 b$ & -915.36899112061 & -129.2751 & 27 \\
\hline 234 & -1183.91374112567 & -218.6292 & 464 \\
\hline
\end{tabular}


Figure 1. Tree diagram of possible pathways generated by ROBIA starting from linear precursor. The numbers in the boxes correspond to the structure numbers in Table 1.
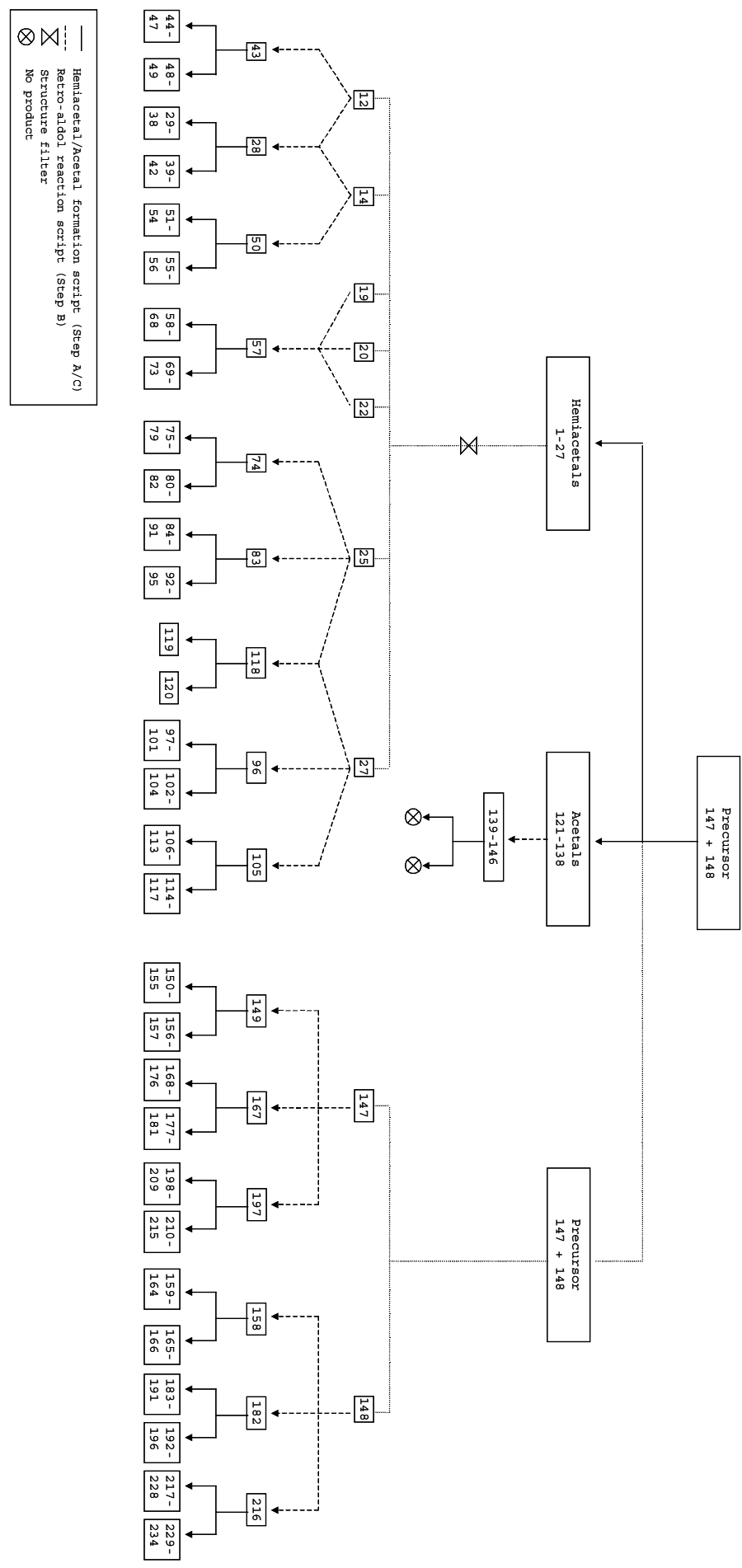